\title{
ASSOCIATION BETWEEN HIP AND KNEE OSTEOARTHRITIS AND ATHEROSCLEROSIS: A SYSTEMATIC REVIEW AND META-ANALYSIS
}

\author{
Marina Barguil Macedo ${ }^{1, \star}$, Victor Matheus Ostrovski Souza Santos ${ }^{1}$, Rosa Maria Rodrigues Pereira ${ }^{1}$, Ricardo Fuller ${ }^{1}$ \\ 1.Universidade de São Paulo, São Paulo (SP), Brazil. \\ *Corresponding author: marina.bm.15@gmail.com
}

\section{BACKGROUND}

An association between osteoarthritis (OA) and atherosclerosis (AT) has long been suggested, with its plausibility based on several guessed commonalities, from shared risk factors to overlapping molecular pathways, given that both conditions represent a state of chronic low-grade inflammation. Meta-analyses of the impact of OA on hard cardiovascular (CV) endpoints have displayed conflicting results, though. In order to discern a more refined association between OA and AT, we performed a systematic review and meta-analysis of studies that focused on OA of weight-bearing joints, i.e., hip and knee, and stratified the results by clinical and subclinical AT.

\section{METHODS}

We searched PubMed, Web of Science, LILACS, and SciELO from inception until August 2020, using the MeSH search terms "osteoarthritis", "aorta", "carotid", "intima-media thickness", "coronary artery disease", "atherosclerosis", "cardiovascular disease", and "death". To appraise the quality of the studies, we applied the Newcastle-Ottawa scale. To assess for heterogeneity, 12 was used. A random-fixed effect model was adopted, and outliers were excluded when detected. Publication bias was ascertained qualitatively by funnel plot, and quantitatively by Egger regression test. Analyses were performed on RStudio version 1.3.1093.

\section{RESULTS}

Our search retrieved 1,335 citations, of which 168 were screened for abstract reading. From those, 127 were selected for full-text reading. After exclusion of duplicates and out of scope articles, a total of 27 studies, comprising 151,187 individuals with OA and 287,444 controls, were included on the narrative synthesis, and 17 on the meta-analysis. All but one study was deemed as of fair or good quality. Fifteen studies found a positive association between $O A$ and AT, the remaining 12 did not reach statistical significance. Four studies subdivided the OA patients by gender, and in half of those a positive association was found exclusively on females. Thirteen studies worked with a subclinical definition of AT, 1 included both subclinical and manifest disease, and the remainder included only manifest disease. Hip and knee OA increased the risk for both subclinical AT (OR $=1.15,95 \% \mathrm{Cl}=1.01-1.31$ ), and CV disease $(\mathrm{OR}=1.13,95 \% \mathrm{Cl}=1.05-1.22)$, but not for $\mathrm{CV}$ death $(\mathrm{OR}=1.08,95 \% \mathrm{Cl}=0.99-1.19)$.

\section{CONCLUSION}

Hip and knee OA are associated with AT. This may have practical implications on the management of OA patients, as a more intensive control of traditional CV risk factors may be advised.

\section{KEYWORDS}

Osteoarthritis, Atherosclerosis, Cardiovascular disease. 